\title{
An Optimal Filtering Algorithm for Table Constraints
}

\author{
Jean-Baptiste Mairy ${ }^{1}$, Pascal Van Hentenryck ${ }^{2}$, and Yves Deville $^{1}$ \\ 1 ICTEAM, Université catholique de Louvain, Belgium \\ \{Jean-Baptiste.Mairy, Yves.Deville\} @uclouvain.be \\ 2 Optimization Research Group, NICTA, University of Melbourne, Australia \\ pvh@nicta.com.au
}

\begin{abstract}
Filtering algorithms for table constraints are constraint-based, which means that the propagation queue only contains information on the constraints that must be reconsidered. This paper proposes four efficient value-based algorithms for table constraints, meaning that the propagation queue also contains information on the removed values. One of these algorithms (AC5TC-Tr) is proved to have an optimal time complexity of $O(r . t+r . d)$ per table constraint. Experimental results show that, on structured instances, all our algorithms are two or three times faster than the state of the art STR2+ and $\mathrm{MDD}^{c}$ algorithms.
\end{abstract}

\section{Introduction}

Domain-consistency algorithms are usually classified as constraint-based (i.e., the propagation queue only contains information on the constraints that must be reconsidered) or value-based (i.e., removed values are also stored in the propagation queue). For table constraints, which have been the focus of much research in recent years, all existing algorithms (except in [14]) are constraint-based. This paper proposes four original value-based algorithms for table constraints, which are all instances of the AC5 generic algorithm. The proposed propagators maintain, for every value of the variables, the index of its first current support in the table. They also use, for each variable of a tuple, the index of the next tuple sharing the same value for this variable. The algorithms differ in their use of information on the validity of the tuples. Three of the proposed algorithms have a time complexity of $O\left(r^{2} \cdot t+r \cdot d\right)$ per table constraint and one of them (AC5TC-Tr) has the optimal time complexity of $O(r \cdot t+r \cdot d)$, where $r$ is the arity of the table, $d$ the size of the largest domain and $t$ the number of tuples in the table. One of the proposed algorithms, AC5TC-Recomp, is the (unpublished) propagator of the Comet system.

Experimental results show that, on structured instances, our algorithms improve upon the state-of-the-art STR2+ [11] and $\mathrm{MDD}^{c}$ [3]: The speedup is between 1.95 and 3.66 over STR2+ and between 1.83 and 4.57 over MDD ${ }^{c}$. Our (theoretically) optimal algorithm is not always the fastest in practice. Interestingly, on purely random tables, our algorithms do not compete with STR2+ and $\mathrm{MDD}^{c}$. Since most real problems are structured, we expect our algorithms to be an interesting contribution to the field. The rest of this paper is organized as follows. Section 2 presents background information and related work. Section 3 describes the first two table-constraint propagators. Section 4 presents our optimal propagator, while Section 5 proposes an efficient variant of our first algorithms. Section 6 describes the experimental results. 


\section{Background}

A CSP $(X, D(X), C)$ is composed of a set of $n$ variables $X=\left\{x_{1}, \ldots, x_{n}\right\}$, a set of domains $D(X)=\left\{D\left(x_{1}\right), \ldots, D\left(x_{n}\right)\right\}$ where $D(x)$ is the set of possible values for variable $x$, and a set of constraints $C=\left\{c_{1}, \ldots, c_{e}\right\}$, with $\operatorname{Vars}\left(c_{i}\right) \subseteq X(1 \leq i \leq e)$. We let $d=\max _{1 \leq i \leq n}\left(\# D\left(x_{i}\right)\right)$, and $D(X)_{x_{i}=a}$ be the set of tuples $\mathbf{v}$ in $D(X)$ with $\mathbf{v}_{i}=a$. Given $Y=\left\{x_{1}, \ldots, x_{k}\right\} \subseteq X$, the set of tuples in $D\left(x_{1}\right) \times \ldots \times D\left(x_{k}\right)$ is denoted by $D(X)[Y]$ or simply $D(Y)$. A support in a constraint $c$ for a variable value pair $(x, a)$ is a tuple $\mathbf{v} \in D(\operatorname{Vars}(c))$ such that $c(\mathbf{v})$ and $\mathbf{v}[x]=a$. The following (Inconsistent and Consistent) sets are useful for specifying domain consistency and propagation methods. Let $c$ be a constraint of a CSP $(X, D(X), C)$ with $y \in \operatorname{Vars}(c)$, and $B(X)$ be some domain.

$$
\begin{aligned}
\operatorname{Inc}(c, B(X)) & =\left\{(x, a) \mid x \in \operatorname{Vars}(c) \wedge a \in D(x) \wedge \forall \mathbf{v} \in B(\operatorname{Vars}(c))_{x=a}: \neg c(\mathbf{v})\right\} \\
\operatorname{Cons}(c, y, b) & =\{(x, a) \mid x \in \operatorname{Vars}(c) \wedge a \in D(x) \wedge \exists \mathbf{v}: \mathbf{v}[x]=a \wedge \mathbf{v}[y]=b \wedge c(\mathbf{v})\} \\
\operatorname{Inc}(c) & =\operatorname{Inc}(c, D(X))
\end{aligned}
$$

A constraint $c$ in a CSP $(X, D(X), C)$ is domain-consistent iff $\operatorname{Inc}(c)=\emptyset$. A CSP $(X, D(X), C)$ is domain-consistent iff all its constraints are domain-consistent.

Table Constraints. Given a set of tuples $T$ of arity $r$, a table constraint $c$ over $T$ holds if $\left(x_{1}, \ldots, x_{r}\right) \in T$. The size $t$ of a table constraint $c$ is its number of tuples, which is denoted by c.length. We assume an implicit ordering of the tuples: $\sigma_{c, i}$ denotes the $i^{t h}$ element of the table in $c$ and $\sigma_{c, i}[x]$ is the value of $\sigma_{c, i}$ for variable $x$. We introduce a top value $T$ (resp. bottom value $\perp$ ) greater (resp. smaller) than any other value. We also introduce a universal tuple $\sigma_{c, \top}$, with $\sigma_{c, \top}[x]=*$ forall $x \in X$ and abuse notations in postulating that $\forall a \in D(x), *=a$. This implies that, for any table $T, \sigma_{c, \top} \in T$. Given a table constraint, we say that a tuple $\sigma$ is allowed if it belongs to the table. A tuple $\sigma$ is valid if all its values belong to the domain of the corresponding variables. To achieve domain consistency, one must at least check the validity of each tuple and, in the worst case, remove all the values from the domains. Hence a domain-consistency algorithm has a complexity $\Omega(r \cdot t+r \cdot d)$ per table constraint in the worst case. An AC5-like algorithm with a complexity $O(r \cdot t+r \cdot d)$ per table constraint is thus optimal. As usual for such algorithms, if a domain-consistency algorithm has a time complexity of $O(f)$, then the time complexity of the aggregate executions of this algorithm along any path in the search tree is also $O(f)$.

Related Work. A lot of research effort has been spent on table constraints. The existing propagators can be categorized in 3 classes: index-based, compression-based, and based on a dynamic table. The index-based approaches use an indexing of the table to speed up its traversal. Examples of such propagators are GAC3_allowed and other constraintbased variants $\left(\mathrm{GAC}_{r m}\right.$ _allowed, GAC2001_allowed) $[10,1,12,6]$. For each variable value pair $(x, a)$, the index data structure has an array of the indexes of the tuples with value $a$ for $x$. The space complexity of the data structure is $O(r \cdot t)$. The time complexity of GAC3_allowed is $O\left(r^{3} \cdot d \cdot t+r \cdot d^{2}\right)$ per table constraint. GAC2001_allowed has a time complexity of $O\left(r^{3} \cdot d \cdot t+r^{2} \cdot t\right)$ per table constraint. Indexing can also be used in 
value-based propagators. In [14], the authors propose a value-based propagator for table constraints implementing GAC6. It uses a structure which indexes, for each variable value pair $(x, a)$ and each tuple, the next tuple in the table with value $a$ for $x$. The space complexity of the data structure is $O(r \cdot d \cdot t)$. This space usage can be reduced by using a data structure called hologram [13]. Another index type, proposed in [7], indexes, for each tuple and variable, the next tuple having a different value for the variable. Compression-based propagators compress the table in a form that allows a fast traversal. One of such compressed forms uses a trie for each variable [7]. Another example of compression-based techniques [3,2] uses a Multi Valued Decision Diagram (MDD) to represent the table more efficiently. During propagation, the tries or MDD are traversed using the current domains to perform the pruning. These algorithms are constraintbased and have a time complexity of $O\left(r^{2} \cdot d \cdot t\right)$ per table constraint. Compression and faster traversal can also be achieved by using compressed tuples, which represent a set of tuples [8,16]. Propagators based on dynamic tables maintain the table by suppressing invalid tuples from it. The STR algorithm [18] and its refined version, STR2 [11], are constraint-based and scan only the previously valid tuples to extract the valid values. The time complexity of STR2 is $O\left(r^{2} \cdot d^{2}+r^{2} \cdot d \cdot t\right)$ per table constraint. The ortool propagator [15] also maintains a dynamic table. It uses a bitset on the tuples of the table to maintain their validity. One bitset per variable value $(x, a)$ is also used for easy access of the tuples with value $a$ for variable $x$. This propagator has a $O(r \cdot d \cdot t)$ time complexity per table constraint.

The AC5 Algorithm. AC5 [19,4] is a generic value-based domain-consistency algorithm. In a value-based approach, information on the removed values is also stored in the queue for the propagation. Specification 1 describes the main methods of AC5 which uses a queue $Q$ of triplets $(c, x, a)$ stating that the domain consistency of constraint $c$ should be reconsidered because value $a$ has been removed from $D(x)$. When a value is removed from a domain, the method enqueue puts the necessary information on the queue. In the postcondition, $Q_{o}$ represents the value of $Q$ at call time. The method post $(\mathrm{c}, \triangle)$ is called once when posting the constraint. It computes the inconsistent values of the constraint $c$ and initializes specific data structures required for the propagation of the constraint. As long as $(c, x, a)$ is in the queue, it is algorithmically desirable to consider that value $a$ is still in $D(x)$ from the perspective of constraint $c$. This is captured by the following definition.

Definition 1. The local view of a domain $D(x)$ wrt a queue $Q$ for a constraint $c$ is defined as $D(x, Q, c)=D(x) \cup\{a \mid(c, x, a) \in Q\}$.

For table constraints, a tuple $\sigma$ is $Q$-valid if all its values belong to $D(X, Q, c)$. The central method of AC5 is the valRemove method, where the set $\triangle$ is the set of values becoming inconsistent because $b$ is removed from $D(y)$. In this specification, $b$ is a value that is no longer in $D(y)$ and valRemove computes the values $(x, a)$ no longer supported in the constraint $c$ because of the removal of $b$ from $D(y)$. Note that values in the queue are still considered in the potential supports as their removal has not yet been reflected in this constraint. The minimal pruning $\triangle_{1}$ only deals with variables and values previously supported by $(y, b)$. However, we give valRemove the possibility of achieving more pruning $\left(\triangle_{2}\right)$, which is useful for table constraints. 


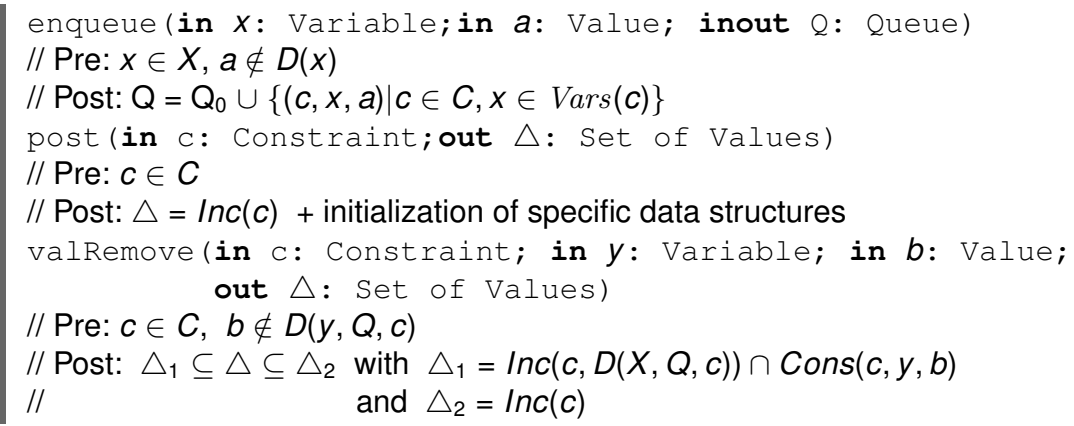

Specification 1: The enqueue, post, and valRemove Methods for AC5

\section{Efficient Value-Based Algorithms for Table Constraints}

Our value-based approaches use a data structure $F S$ memorizing first supports. Intuitively $F S[x, a]$ is the index of the first Q-valid support of the variable value pair $(x, a)$. To speed up the table traversal, our algorithms use a second data structure called next that links all the elements of the table sharing the same value for a given variable. The next data structure is semantically equivalent to the index of [12]. More formally, for a given table constraint $c, F S$ and next satisfy the following invariant (called FS-invariant) before dequeuing an element from $Q$.

$$
\begin{aligned}
& \forall x \in \operatorname{Vars}(c) \forall a \in D(x, Q, c): F S[x, a]=i \Leftrightarrow \\
& \quad \sigma_{c, i}[x]=a \wedge i \neq \top \wedge \sigma_{c, i} \in D(\operatorname{Vars}(c), Q, c) \wedge \\
& \forall j<i: \sigma_{c, j}[x]=a \Rightarrow \sigma_{j} \notin D(\operatorname{Vars}(c), Q, c) \\
& \forall x \in \operatorname{Vars}(c) \forall 1 \leq i \leq \text { c.length }: \operatorname{next}[x, i]=\operatorname{Min}\left\{j \mid i<j \wedge \sigma_{c, j}[x]=\sigma_{c, i}[x]\right\}
\end{aligned}
$$

The next data structure, illustrated in Figure 1, is static as it does not depend on the domain of the variables. However, FS must be trailed during the search. Methods postTC and valRemoveTC are given in Algorithms 1 and 2. They use the seekNextSupportTC method (Algorithm 3) which searches the next Q-valid tuple. Abstract method isQValidTC (c, i) tests whether $\sigma_{c, i}$ is Q-valid (i.e., $\sigma_{c, i} \in$ $D(X, Q, c))$ and can be implemented in many ways. One simple way is to record the Qvalidity of tuples in some data structure, initialized in method initspecstruct TC and updated in method setQInvalidTC. Method postTC initializes the $F S$ and next data structures and returns the set of inconsistent values. Method valRemoveTC has only to consider the tuples in the next chain starting at $F S[y, b]$. When one of these tuples $\sigma_{c, i}$ is the first support of an element $a=\sigma_{c, i}[x]$, a new support $F S[x, a]$ must be found. If such a support does not exist, then $(x, a)$ belongs to the set $\triangle_{1}$. Method valRemoveTC thus computes the set $\triangle_{1}$ and maintains the FS-invariant.

Not considering initspecStruct TC, method post TC has a time complexity of $O(r t+r d)$. After the post TC method, the domain size of $x$ is $O(t)$. We now establish the complexity of all executions of valRemoveTC for a given table constraint, 


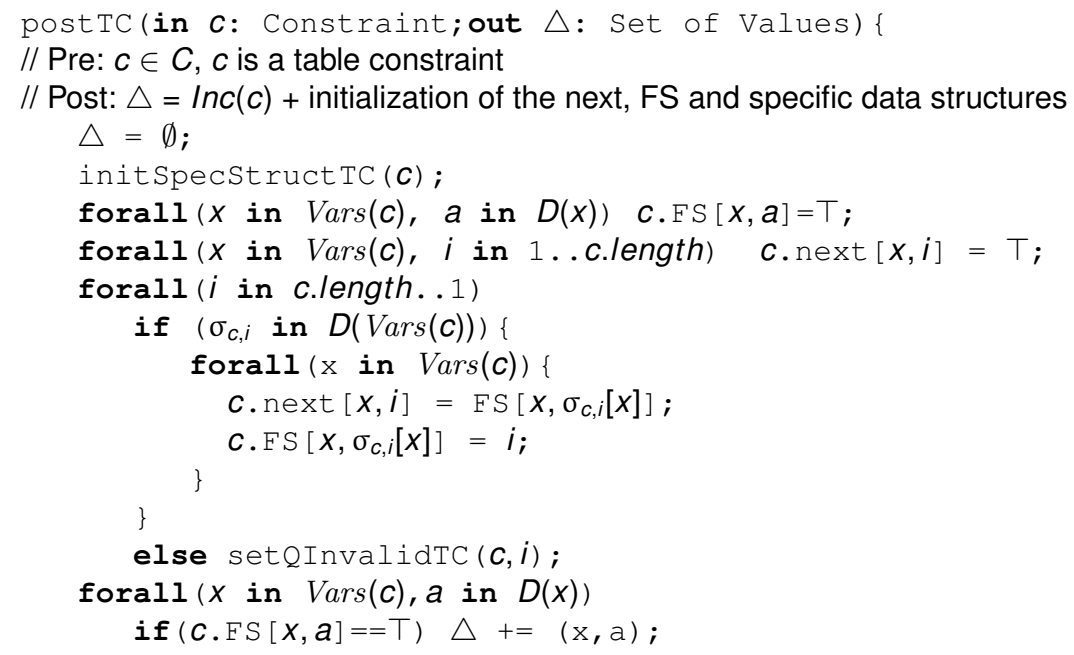

Algorithm 1: Method post TC for Table Constraints

assuming this table constraint is one of the constraints of the CSP on which domain consistency is achieved. Consider first all executions of valRemoveTC without line 13. For a given variable $y$, these executions follow the different next chains of the variable $y$. The chains for all values of $y$ have a total number of $t$ elements. The complexity of lines 9-16 (without line 13) is $O(r)$. Since the table has $r$ variables, the complexity of all valRemoveTC executions during the fixed point (without line 13) is thus $O\left(r^{2} \cdot t\right)$, assuming a $O(1)$ complexity of setQInvalidTC. Consider now all executions of line 13 in valRemoveTC for a variable $x$. Since line 13 always increases the value of $F S[x, a]$ in the next chain of $(x, a)$, we have a global complexity of $O(V \cdot t)$ for the variable $x$, where $V$ is the time complexity of isQValidTC. All executions of line 13 in valRemoveTC thus take time $O(V \cdot r \cdot t)$. The time complexity of all executions of valRemoveTC is then $O\left(r^{2} \cdot t+V \cdot r \cdot t\right)$. Even with a $O(1)$ the time complexity of isQValidTC, the algorithm is not optimal but it turns out to be more efficient than state-of-the-art algorithms on different classes of problems. The AC5 algorithm with the postTC and valRemoveTC implementation for table constraint is called AC5TC (AC5 for Table Constraints).

Proposition 1. Assuming that init SpecStruct TC and set QInvalidTC have a time complexity of $O(r \cdot t+r \cdot d)$ and $O(1)$ respectively and allow a correct implementation of isQVaI idTC to have a complexity of $O(r)$, then AC5TC is correct and has a time complexity of $O\left(r^{2} \cdot t+r \cdot d\right)$ per table constraint.

We now present two implementations of AC5TC. They differ in the implementations of methods isQValidTC, setQInvalidTC and init SpecStructTC. AC5TCBool, the first implementation of AC5TC, is shown in Algorithm 4. It uses a data structure is QValid $[i]$ to record the Q-validity of the element $\sigma_{c, i}$. It satisfies invariant 


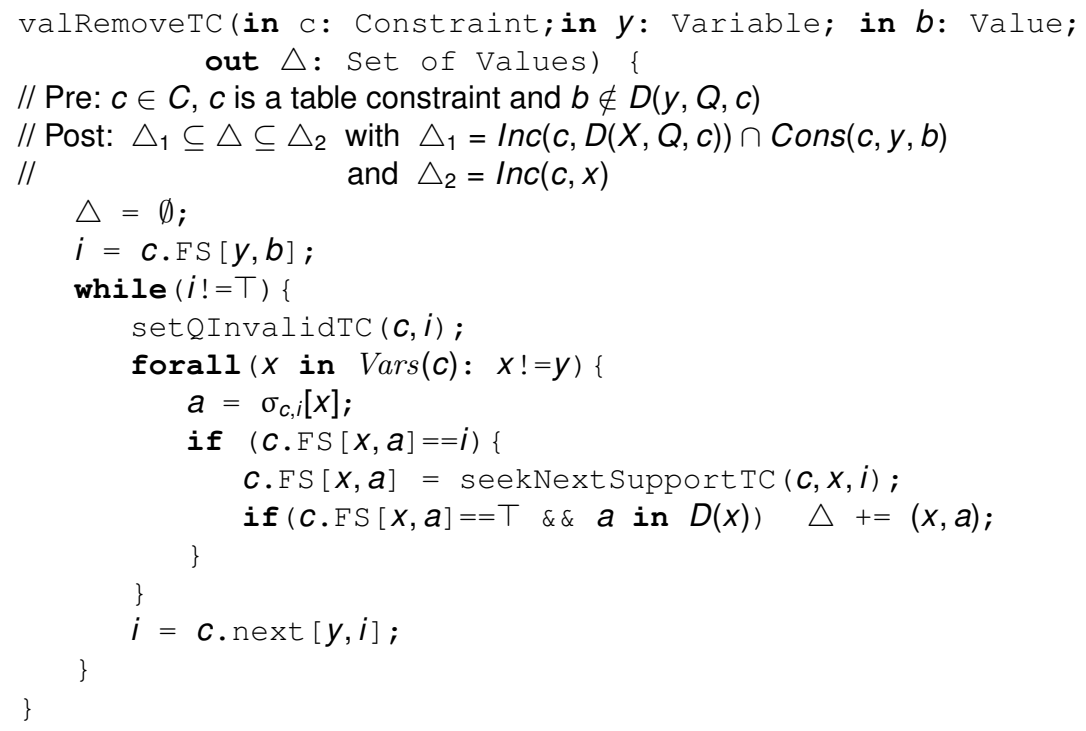

Algorithm 2: Method valRemoveTC for Table Constraints.

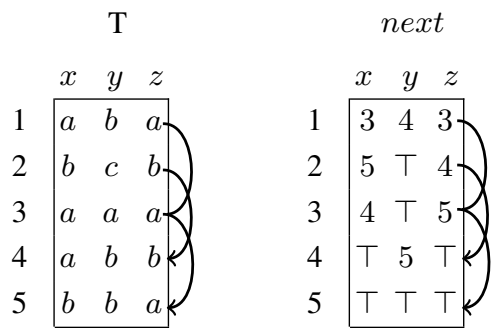

Fig. 1: Example of a next data structure of a table T (arrow pointers for variable $z$ only).

is QValid $[i] \Leftrightarrow \sigma_{c, i} \in D(X, Q, c)$ before dequeuing an element from $Q(1 \leq i \leq$ c.length). The data structure must be trailed as it depends on the domains. The methods for Q-validity are given in Algorithm 4. As the methods isQValidTC-Bool is correct, AC5TC-Bool is correct. The time complexity of isQValidTC-Bool and setQInvalidTC-Bool is $O(1)$ and initspecStructTC-Bool is $O(t)$. The time complexity of AC5TC-Bool is then $O\left(r^{2} \cdot t+r \cdot d\right)$ per table constraint.

AC5TC-Bool must trail the is QValid boolean array. We now propose an implementation that only trails one integer, building upon an idea in STR and STR2 [18,11]. The implementation simply keeps invalid elements at the end of the table, with a single variable size representing the boundary between valid (before position size) and invalid elements (after position size). When an element becomes invalid, it is swapped with the element at position size and size is decremented by one. The size variable must be trailed but the table does not need to: The valid elements are automatically restored, albeit at a different position in the table. This is sometimes called semantic backtracking [20]. Instead of swapping tuples, our implementation uses two arrays 


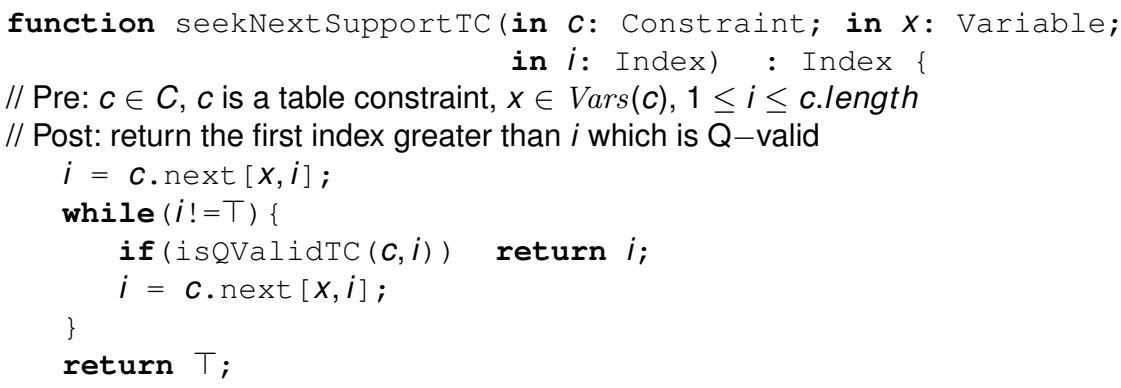

Algorithm 3: Function seekNext Support TC for Table Constraints.

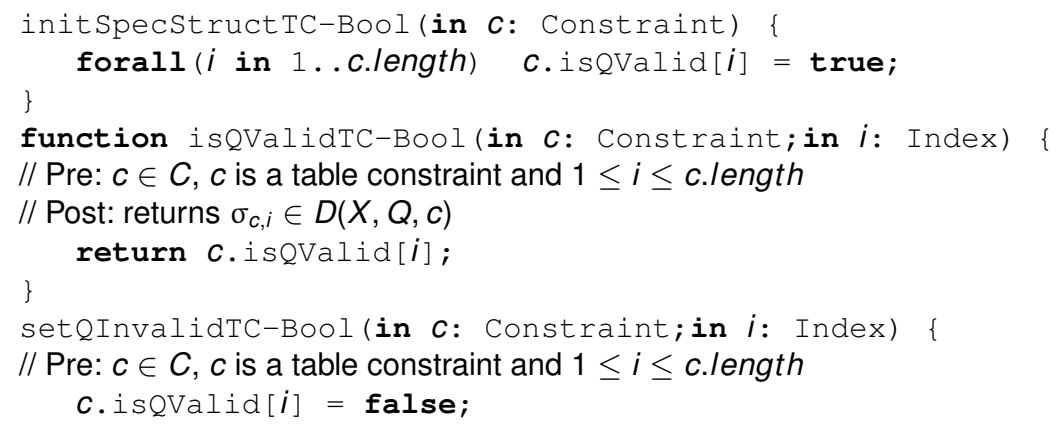

Algorithm 4: Implementation of the specific methods of AC5TC-Bool

Map and Dyn that give the virtual position of the tuples and the positions of the virtual tuples in the table. These arrays do not have to be trailed. For a given table constraint $c$, the data structures satisfy the following invariants before dequeuing an element from $Q$ $(1 \leq i \leq$ c.length $): \operatorname{Map}[i] \leq \operatorname{size} \Leftrightarrow \sigma_{i} \in D(X, Q, c) \wedge \operatorname{Dyn}[\operatorname{Map}[i]]=i$

The implementations are given in Algorithm 5 and the algorithm is called AC5TCCutOff. The time complexity of isQValidTC-Cutoff and setQInvalidTC-Cutoff is $O(1)$ and initspecstruct TC-Cutoff takes time $O(t)$. The time complexity of AC5TC-CutOff is thus $O\left(r^{2} \cdot t+r \cdot d\right)$.

\section{An Optimal Algorithm}

In method valRemoveTC, executions of the seekNext Support TC (line 12 of Algorithm 2) take $O(r \cdot t)$ assuming isQValidTC takes constant time. However, the method revisits Q-invalid tuples because the next data structure is static. To remedy this situation, the idea is to make the next data structure dynamic and to always ensure that the element following a Q-valid element in a next chain is also Q-valid. This avoids unnecessary Q-validity checks and can be easily implemented using a doubly-linked list. 


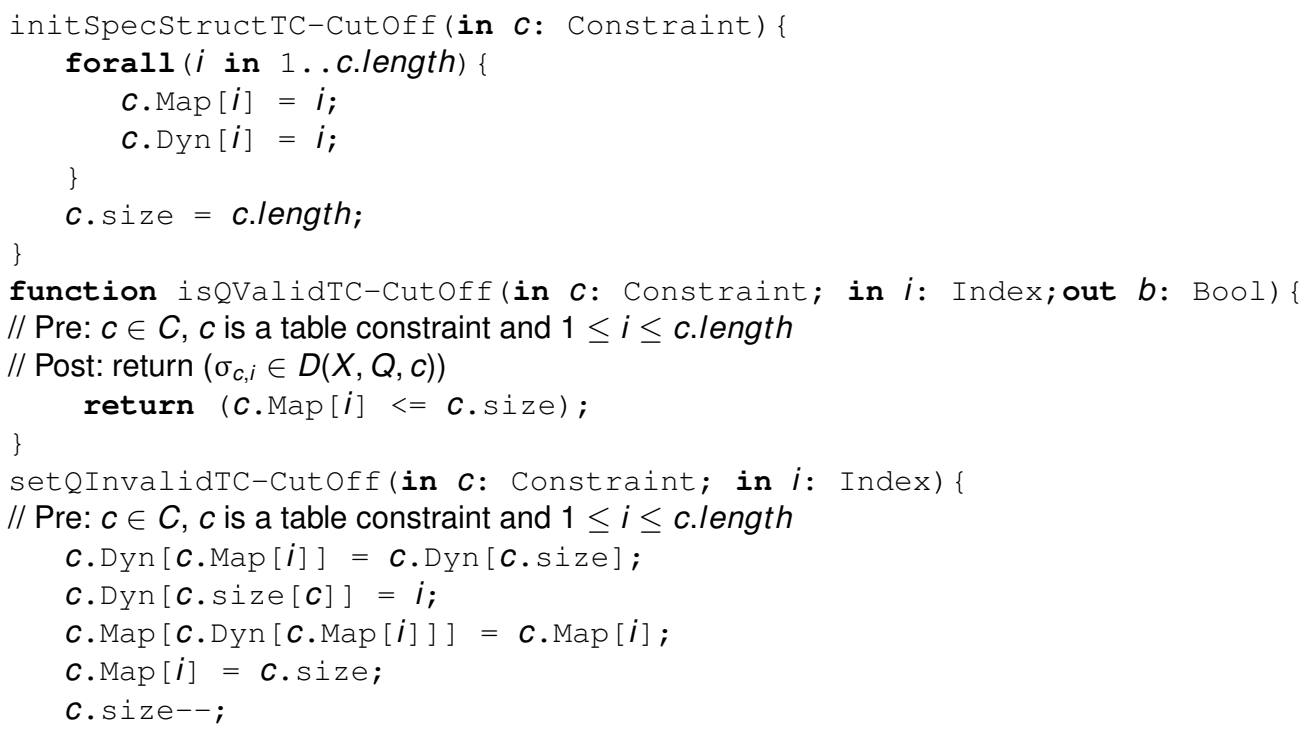

Algorithm 5: Implementation of the specific methods of AC5TC-CutOff

More formally, for a given table constraint $c$, the data structure satisfies the following invariant before dequeuing an element from $Q$

$$
\begin{aligned}
& \forall x \in \operatorname{Vars}(c) \forall 1 \leq i \leq \text { c.length }: \\
& n \operatorname{ext} \operatorname{Tr}[x, i]=\operatorname{Min}\left\{j \mid i<j \wedge \sigma_{c, j}[x]=\sigma_{c, i}[x]\right. \\
& \left.\wedge\left(\sigma_{c, i} \in D(X, Q, c) \Rightarrow \sigma_{c, j} \in D(X, Q, c)\right)\right\} \\
& \operatorname{pred} \operatorname{Tr}[x, \operatorname{next} \operatorname{Tr}[x, i]]=i
\end{aligned}
$$

The nextTr and predTr data structures should be trailed as they depend on the current domains. The algorithm also uses the $F S$ data structure with its original invariant. No other data structures are necessary.

Methods postTC-Tr and valRemove TC-Tr are given in Algorithms 6 and 7. Method postTC-Tr now initializes predTr as well. Method valRemoveTC-Tr $(c, y, b)$ does not need to search for a support as the next element in the nextTr chain is necessarily Q-valid. However, if the first support for $(x, a)$ is before $F S[y, b]$ (it cannot be after because of the FS invariant), nextTr and predTr must be updated to ensure that the new invalid tuples are no longer in the next chains. It will thus never be visited twice. Method valRemoveTC-Tr computes the set $\triangle_{1}$ and maintains the invariants on $F S$ and on nextTr / predTr.

Method postTC-Tr has a time complexity of $O(r \cdot t+r \cdot d)$. We establish the complexity of all executions of valRemoveTC-Tr for a given table constraint during the fixed point algorithm, assuming the presence of other constraints on which domain consistency is also enforced. We first show that all executions of lines 7 and 21 lead to 


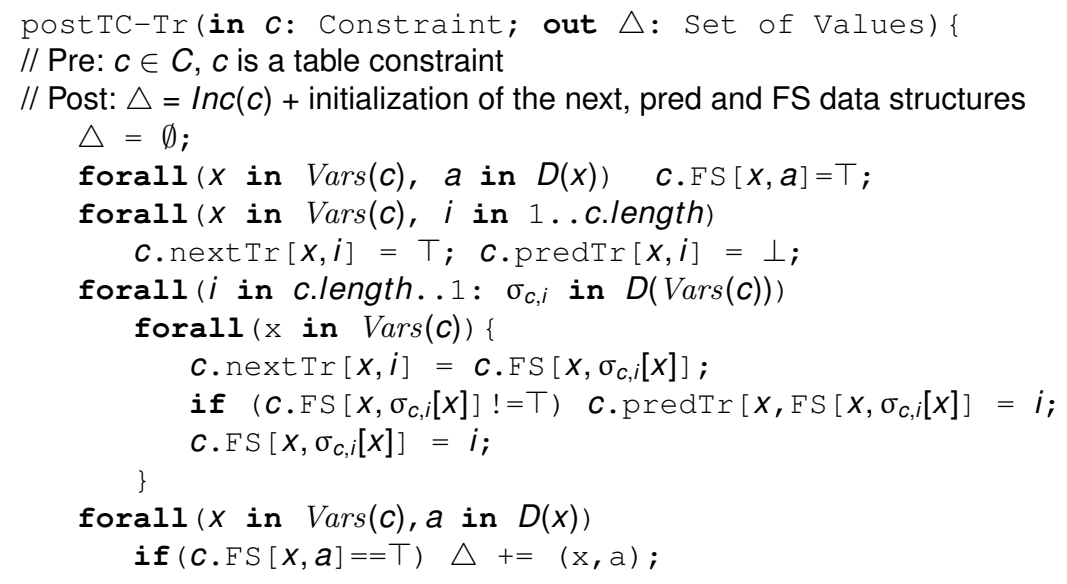

Algorithm 6: An optimal post TC-Tr method for Table Constraints

different values of $i$ in $\{1, \ldots, t\}$ (except when $i==\top$ ). By the FS invariant, we never have $i==\top$ at line 7 . For a given value $i \neq \top$, by the FS invariant, $F S[x, a]==i$ or $F S[x, a]<i$ holds at line 11. If $F S[x, a]==i, F S[x, a]$ is incremented and the tuple $i$ will never be reconsidered by removing $a$ from $D(x)$. If $F S[x, a]<i$, the tuple $i$ is removed from the nextTr chain and will never be reconsidered. This holds for all variables $x \neq y$. Hence the tuple $i$ will never be reconsidered in future executions of valRemoveTC-Tr. Hence, lines 9-20 are executed $O(t)$ times. Since the complexity of lines 9-20 is $O(r)$, the aggregate complexity of all executions of valRemoveTC-Tr is $O(r \cdot t)$. The AC5 algorithm with the post TC-Tr and valRemoveTC-Tr implementation for table constraint is called AC5TC-Tr (AC5 for Table Constraints with Trailing).

Proposition 2. AC5TC-Tr is correct and has an optimal time complexity of $O(r \cdot t+r \cdot d)$ per table constraint.

\section{A Variation Based on Recomputation}

We now propose a variation of the AC5TC algorithm, called AC5TC-Recomp, that does not require any data structure to maintain the Q-validity of tuples. AC5TC-Recomp is the (unpublished) table constraint algorithm of the Comet system. It replaces the Qvalidity test by a function isValidTC that tests the validity of the tuples. The straightforward implementation of the isvalid function is given in Algorithm 8. Method init SpecStruct TC and setQInvalidTC are just empty. Since AC5TC-Recomp tests validity instead of Q-validity, method valRemoveTC must be slightly modified; the test $a \in D(x)$ should be moved from line 14 to line 10 which becomes ${ }^{3}$

forall ( $x$ in $\operatorname{Vars}(c): x !=y \quad \& \& \sigma_{c, i}[x]$ in $\left.D(x)\right)\{$

\footnotetext{
${ }^{3}$ This modification also maintains the correctness of our generic AC5TC algorithm but requires a more sophisticated FS-invariant. With this change, our earlier algorithms would have the same theoretical complexity but are less efficient in practice.
} 


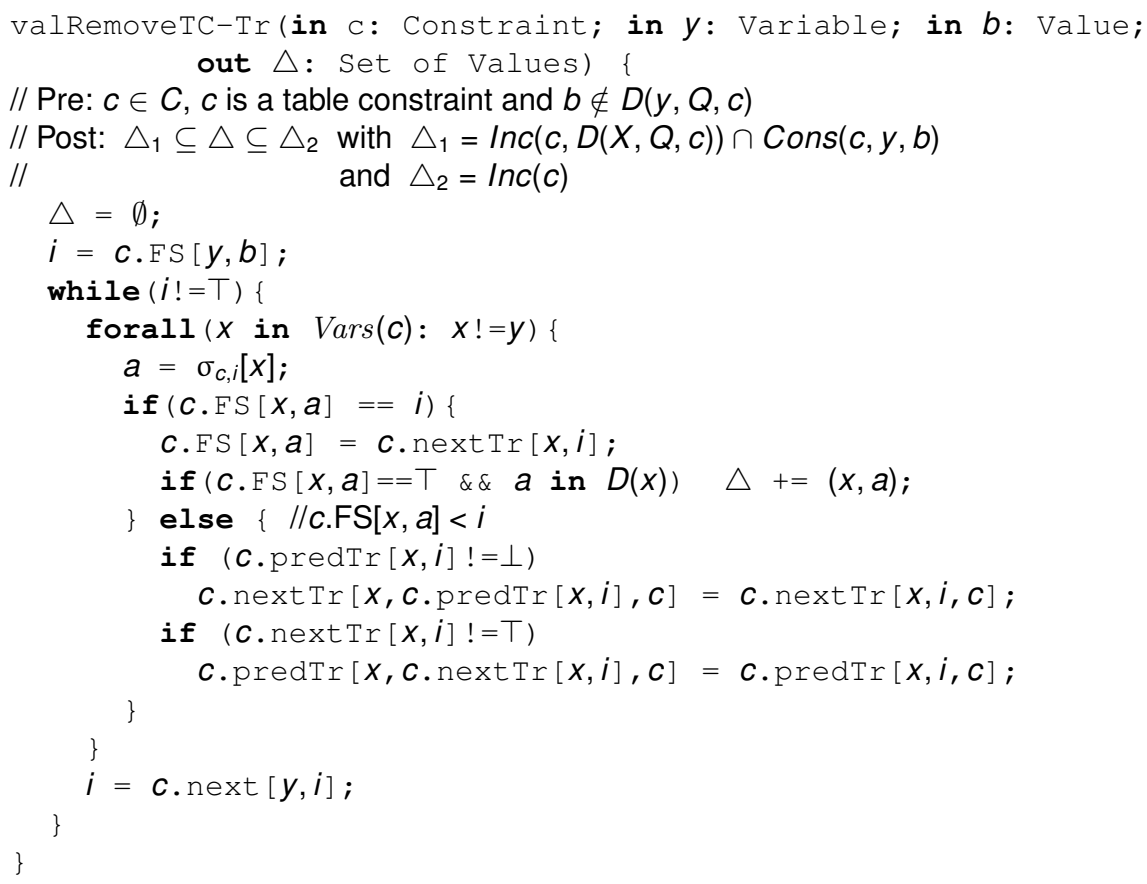

Algorithm 7: An Optimal valRemoveTC-Tr method for Table Constraints

This modified version of valRemoveTC exploits the flexibility of its specification by computing a set $\triangle$ between $\triangle_{1}$ and $\triangle_{2}$. AC5TC-Recomp has a runtime complexity of is $O\left(r^{2} \cdot t+r \cdot d\right)$ and per table constraint and improves state-of-the-art algorithms on some classes of problems.

\section{Experimental Results}

All proposed algorithms have been implemented on top of Comet, including AC5TCRecomp. For comparison, classical constraint-based algorithms have also been implemented on top of Comet. The GAC3-Allowed algorithm has been chosen because it is the standard GAC3 algorithm for the table constraints [10]. The two state-of-the-art methods were also reimplemented: The STR2+ algorithm from [11] and the MDD ${ }^{c}$ algorithm from [3]. They are respectively called STR and MDD in the experimental results. All experiments were conducted on an Intel Xeon 2.53GHz using Comet 2.1.1. The algorithms are compared within a MAC search. This section presents results on fully random instances, on the geometric problem, on Langford problem, and on the Traveling Salesman Problem.

For each instance set, the experimental results report the mean execution times in seconds (totTime), the mean "posting" times in seconds (post T), the number of propagator calls (nProp), the percentage to the best with respect to execution time (\%best), the mean of percentage to the best algorithm in terms of execution time ( $\mu \%$ best), the num- 


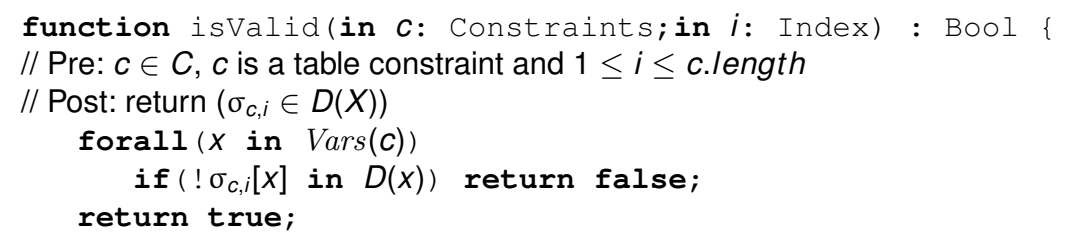

Algorithm 8: The isValid Function of AC5TC-Recomp.

ber of validity checks (valChk), Q-validity checks (QvalChk), and the number of pointers followed ( $p$ Follow). The difference between the \%best and $\mu \%$ best is the following: for \%best, the execution times are averaged before computing the quantity. There is thus one best algorithm. For $\mu \%$ best, the percentages are computed instance by instance and aggregated with a geometrical mean at the end. This measure takes into account that different instances may have different best algorithms. The $\mu \%$ best measure uses a geometrical mean as suggested in [5]. The last reported quantity, pFollow, has different meanings for different algorithms. For GAC3-Allowed, it corresponds to the number of times the tuples are accessed. For the AC5TC algorithms, it is defined as the number of times the next or next $T r$ structures are used to traverse the table. For MDD, it corresponds to the number of edges followed in the MDD structure. Although referring to different quantities, pFollow is useful for comparing the behavior of the propagators as it reflects the usage of their specific structures.

Random Instances These instances contain random table constraints of random scope generated by the RD-model [22]. Parameters are chosen to generate instances close to the phase transition, using Theorems 1 and 2 from [22]. The instances have 10 variables, a uniform domain size of 10 , and 15 table constraints of arity 5 . The expected number of tuples in each table is thus 20000. 10 instances were generated with those settings. The search strategy is the dom heuristic with lexicographic value ordering.

\begin{tabular}{lrrrrrrrr}
\hline propagator & totTime & postT & nProp & $\%$ best & $\mu \%$ best & valChk & QvalChk & pFollow \\
\hline GAC3-Allowed & 3000 & 1.5 & $614 \mathrm{k}$ & 2725 & 2660 & $523 \mathrm{M}$ & 0 & $523 \mathrm{M}$ \\
AC5TC-Bool & 4636 & 1.0 & $2.8 \mathrm{M}$ & 4211 & 4070 & $19 \mathrm{k}$ & $257 \mathrm{M}$ & $481 \mathrm{M}$ \\
AC5TC-CutOff & 3991 & 0.8 & $2.8 \mathrm{M}$ & 3626 & 3538 & $19 \mathrm{k}$ & $257 \mathrm{M}$ & $481 \mathrm{M}$ \\
AC5TC-Tr & 994 & 5.2 & $2.8 \mathrm{M}$ & 903 & 930 & $19 \mathrm{k}$ & 0 & $16 \mathrm{M}$ \\
AC5TC-Recomp & 3874 & 0.8 & $2.4 \mathrm{M}$ & 3519 & 3357 & $98 \mathrm{M}$ & 0 & $305 \mathrm{M}$ \\
STR2 & 483 & 0.7 & $614 \mathrm{k}$ & 439 & 455 & $22 \mathrm{M}$ & 0 & 0 \\
MDD & $\mathbf{1 1 0}$ & 12.4 & $614 \mathrm{k}$ & 100 & 100 & 0 & 0 & $12 \mathrm{M}$ \\
\hline
\end{tabular}

Table 1: Results of the propagators on fully random instance set (times in seconds)

Table 1 summarizes the results, which remain similar for other parameter settings. The standard STR2 and MDD algorithms outperform our value-based propagators. Observe the large number of validity checks of AC5TC-Recomp and Q-validity checks of 
AC5TC-Bool and AC5TC-CutOff, as well as the number of times they follow a pointer. AC5TC-Tr, the best value-based propagator, follows far less pointers than our other propagators because it does not follow pointers to a previously inspected tuple. Due to the lack of structure of the constraint set, the first three AC5TC propagators check multiple times the same tuples. Also, those random instances have large tables, which makes the cost of the trailable next Tr structure in AC5TC-Tr too high.

The Geometric Problem Instances of the geometric problem are random instances generated following a specific structure proposed by Rick Wallace [21]. Each variable is randomly placed in the unit square. A fixed distance (less than $\sqrt{2}$ ) is randomly chosen. For each pair of variables $(x, y)$, if the distance between their associated points is less than or equal to this fixed distance, the arc $(x, y)$ is added to the constraint graph. Constraint relations are then created like in fully random CSP instances. We use the instance set from [9] which counts 100 instances. The search strategy uses the heuristic dom/deg with lexicographic value ordering. A timeout of 5 minutes has been used. The quantity \%solv gives the percentage of solved instances.

\begin{tabular}{lrrrrrrrrr}
\hline propagator & totTime & postT & nProp & $\%$ best & $\mu \%$ best & $\%$ solv valChk & QvalChk pFollow \\
\hline GAC3-Allowed & 10.1 & 0.3 & $288 \mathrm{k}$ & 128 & 138 & 86 & $28 \mathrm{k}$ & 0 & $28 \mathrm{k}$ \\
AC5TC-Bool & 12.5 & 0.3 & $867 \mathrm{k}$ & 158 & 159 & 84 & 300 & $25 \mathrm{k}$ & $50 \mathrm{k}$ \\
AC5TC-CutOff & 10.8 & 0.2 & $867 \mathrm{k}$ & 137 & 131 & 86 & 300 & $25 \mathrm{k}$ & $50 \mathrm{k}$ \\
AC5TC-Tr & 9.6 & 0.8 & $867 \mathrm{k}$ & 122 & 200 & 87 & 300 & 0 & $13 \mathrm{k}$ \\
AC5TC-Recomp & $\mathbf{7 . 9}$ & 0.2 & $831 \mathrm{k}$ & 100 & 100 & 87 & $6 \mathrm{k}$ & 0 & $29 \mathrm{k}$ \\
STR2 & 24.9 & 0.3 & $288 \mathrm{k}$ & 315 & 316 & 82 & $26 \mathrm{k}$ & 0 & 0 \\
MDD & 14.7 & 1.6 & $288 \mathrm{k}$ & 186 & 337 & 86 & 0 & 0 & $65 \mathrm{k}$ \\
\hline
\end{tabular}

Table 2: Results of the propagators on the geom instances (times in seconds)

Table 2 presents the experimental results. The quantities are computed on instances for which none of the techniques timeout. All our propagators outperform the state-of-theart STR and MDD. AC5TC-Tr and AC5TC-Recomp are also better than the classical AC3-Allowed. AC5TC-Recomp is the fastest on these instances. which are relatively easy and contain only binary tables. Checking the validity (not costly for binary tables) allows AC5TC-Recomp to follow less pointers than AC5TC-Bool and AC5TC-CutOff by performing longer jumps in the table. The cost of the data structures in AC5TC-Tr is too expensive and outweights its benefits. The large difference between \%best and $\mu \%$ best for AC5TC-Tr is due to the easiest instances, where the propagator is even more disadvantaged due to the cost of its data structures. AC5TC-Recomp also performs less validity checks than STR2 and the number of pointers followed by our propagators are less than those of MDD.

Langford Number Problem Langford number problem $L(k, n)$ amounts to arranging $k$ sets of numbers 1 to $n$ into a sequence of numbers, so that each occurrence of a number $m$ is $m$ numbers apart from its previous occurrence. Those problems are modeled with 
binary (positive) table constraints only. The search strategy used is dom/deg with lexicographic value ordering. Problems where all the propagators take more than 5 minutes are removed from the sets. For $k=2,12$ instances are used: $n \in\{5 . .12,15,16,19,20\}$, for $k=3,8$ instances: $n \in\{3 . .10\}$ and for $k=4,9$ instances: $n \in\{3 . .11\}$. The results for $k$ of 2, 3 and 4 can respectively be found in Table 3 .

\begin{tabular}{|c|c|c|c|c|c|c|c|c|}
\hline propagator & totTime & postT & nProp & \%best & $\mu \%$ best & valChk & QvalChk & pFollow \\
\hline & \multicolumn{8}{|c|}{$k=2$} \\
\hline GAC3-Allowed & 16.3 & 0.6 & $1 \mathrm{M}$ & 173 & 172 & $166 \mathrm{k}$ & 0 & $166 \mathrm{k}$ \\
\hline AC5TC-Bool & 18.6 & 0.8 & $2 \mathrm{M}$ & 197 & 182 & 576 & $178 \mathrm{k}$ & $316 \mathrm{k}$ \\
\hline AC5TC-CutOff & 16.8 & 0.5 & $2 \mathrm{M}$ & 178 & 147 & 576 & $178 \mathrm{k}$ & $316 \mathrm{k}$ \\
\hline AC5TC-Tr & 9.4 & 2.5 & $2 \mathrm{M}$ & 100 & 260 & 576 & 0 & $42 \mathrm{k}$ \\
\hline AC5TC-Recomp & 10.1 & 0.4 & $2 \mathrm{M}$ & 107 & 106 & $27 \mathrm{k}$ & 0 & $154 \mathrm{k}$ \\
\hline STR2 & 26.7 & 1.3 & $1 \mathrm{M}$ & 283 & 342 & $46 \mathrm{k}$ & 0 & 0 \\
\hline \multirow[t]{2}{*}{ MDD } & 26.6 & 3.7 & $1 \mathrm{M}$ & 282 & 517 & 0 & 0 & $307 \mathrm{k}$ \\
\hline & \multicolumn{8}{|c|}{$k=3$} \\
\hline GAC3-Allowed & 2.5 & 0.3 & $75 \mathrm{k}$ & 162 & 148 & $12 \mathrm{k}$ & 0 & $12 \mathrm{k}$ \\
\hline AC5TC-Bool & 3.5 & 0.3 & $242 \mathrm{k}$ & 227 & 184 & 380 & $10 \mathrm{k}$ & $21 \mathrm{k}$ \\
\hline AC5TC-CutOff & 2.5 & 0.2 & $242 \mathrm{k}$ & 163 & 147 & 380 & $10 \mathrm{k}$ & $21 \mathrm{k}$ \\
\hline AC5TC-Tr & 2.2 & 0.9 & $242 \mathrm{k}$ & 140 & 198 & 380 & 0 & $4 \mathrm{k}$ \\
\hline AC5TC-Recomp & 1.5 & 0.2 & $239 \mathrm{k}$ & 100 & 107 & $2 \mathrm{k}$ & 0 & $12 \mathrm{k}$ \\
\hline STR2 & 3.7 & 0.6 & $75 \mathrm{k}$ & 240 & 243 & $5 \mathrm{k}$ & 0 & 0 \\
\hline \multirow[t]{2}{*}{ MDD } & 3.9 & 1.5 & $75 \mathrm{k}$ & 249 & 360 & 0 & 0 & $22 \mathrm{k}$ \\
\hline & \multicolumn{8}{|c|}{$k=4$} \\
\hline GAC3-Allowed & 23.4 & 1.3 & $419 \mathrm{k}$ & 137 & 155 & $19 \mathrm{k}$ & 0 & $19 \mathrm{k}$ \\
\hline AC5TC-Bool & 42.5 & 1.6 & $1.6 \mathrm{M}$ & 250 & 215 & 677 & $20 \mathrm{k}$ & $36 \mathrm{k}$ \\
\hline AC5TC-CutOff & 29.8 & 1.0 & $1.6 \mathrm{M}$ & 175 & 157 & 677 & $20 \mathrm{k}$ & $36 \mathrm{k}$ \\
\hline AC5TC-Tr & 21.8 & 5.0 & $1.6 \mathrm{M}$ & 128 & 254 & 677 & 0 & $5 \mathrm{k}$ \\
\hline AC5TC-Recomp & 17.0 & 0.8 & $1.58 \mathrm{M}$ & 100 & 100 & $3 \mathrm{k}$ & 0 & $18 \mathrm{k}$ \\
\hline STR2 & 33.2 & 3.3 & $419 \mathrm{k}$ & 195 & 277 & $10 \mathrm{k}$ & 0 & 0 \\
\hline MDD & 31.2 & 7.3 & $419 \mathrm{k}$ & 183 & 392 & 0 & 0 & $35 \mathrm{k}$ \\
\hline
\end{tabular}

Table 3: Experimental Results on Langford instances (times in seconds)

Except for AC5TC-Bool on the $k=4$ set of instances, all our propagators improve the state-of-the-art STR and MDD. AC5TC-Tr and AC5TC-Recomp are also better than the classical AC3-Allowed. AC5TC-Tr is the fastest propagator for $k=2$ and AC5TCRecomp is the fastest on the other instance sets. Observe that the number of followed pointers is globally higher for the first instance set $(k=2)$, due to inclusion of instances 
with larger $n$. The number of calls to the propagators during the search is also higher for the $k=2$ set. This suggests that AC5TC-Tr requires harder instances (found in the $k=2$ set) for amortizing the cost of its data structures. For the last two sets, AC5TC-Tr is the second fastest propagator in terms of mean solving time. AC5TC-Tr is closer to AC5TC-Recomp on the $k=4$ instance set. The $k=4$ instance set includes an instance for which $n=11$ (for $k=3: n \leq 10$ ). Here again, the large difference between \%best and $\mu \%$ best for AC5TC-Tr can be attributed to the easiest instances.

Traveling Salesman Problems We conclude with results of the propagators on the Traveling Salesman Problem (TSP) constraint satisfaction instances. We used the set of instances $t s p-20$ and $t s p-25$ [9]. Those structured instances are composed of very different table constraints. Their arity varies between 2 and 3 and they may count up to 20000 tuples but also as few as 20. The variables also have quite different domains: Some have small domains, while others feature domains containing up to 1000 values. There are 61 variables and 230 table constraints in $t s p-20$ instances. The $t s p-25$ instances count 76 variables and 350 constraints. The negative table constraints found in those instances have been transformed into positive ones. The search strategy used here is dom/deg with lexicographic value ordering.

\begin{tabular}{lrrrrrrrr}
\hline propagator & totTime & postT & nProp & $\%$ best & $\mu \%$ best & valChk & QvalChk & pFollow \\
\hline GAC3-Allowed & 797 & 1.7 & $6.7 \mathrm{M}$ & 733 & 587 & $11 \mathrm{M}$ & 0 & $11 \mathrm{M}$ \\
AC5TC-Bool & 186 & 0.8 & $21.2 \mathrm{M}$ & 171 & 187 & $2 \mathrm{k}$ & $1 \mathrm{M}$ & $2 \mathrm{M}$ \\
AC5TC-CutOff & 153 & 0.5 & $21.2 \mathrm{M}$ & 141 & 144 & $2 \mathrm{k}$ & $1 \mathrm{M}$ & $2 \mathrm{M}$ \\
AC5TC-Tr & 120 & 3.3 & $21.2 \mathrm{M}$ & 111 & 164 & $2 \mathrm{k}$ & 0 & $466 \mathrm{k}$ \\
AC5TC-Recomp & $\mathbf{1 0 9}$ & 0.3 & $20.9 \mathrm{M}$ & 100 & 104 & $391 \mathrm{k}$ & 0 & $1 \mathrm{M}$ \\
STR2 & 398 & 1.4 & $6.7 \mathrm{M}$ & 366 & 353 & $803 \mathrm{k}$ & 0 & 0 \\
MDD & 456 & 19.0 & $6.7 \mathrm{M}$ & 419 & 769 & 0 & 0 & $7 \mathrm{M}$ \\
\hline \multicolumn{7}{c}{ Table 4: Results of the propagators for instance set TSP-20 (times in seconds) }
\end{tabular}

\begin{tabular}{lrrrrrrrr}
\hline propagator & totTime & postT & nProp & \%best & $\mu \%$ best & valChk & QvalChk & pFollow \\
\hline GAC3-Allowed & 6607 & 2.4 & $73 \mathrm{M}$ & 606 & 509 & $23 \mathrm{M}$ & 0 & $23 \mathrm{M}$ \\
AC5TC-Bool & 2625 & 1.3 & $198 \mathrm{M}$ & 241 & 233 & $2 \mathrm{k}$ & $11 \mathrm{M}$ & $19 \mathrm{M}$ \\
AC5TC-CutOff & 1937 & 0.7 & $198 \mathrm{M}$ & 178 & 175 & $2 \mathrm{k}$ & $11 \mathrm{M}$ & $19 \mathrm{M}$ \\
AC5TC-Tr & $\mathbf{1 0 8 9}$ & 5.2 & $198 \mathrm{M}$ & 100 & 100 & $2 \mathrm{k}$ & 0 & $3 \mathrm{M}$ \\
AC5TC-Recomp & 1315 & 0.5 & $196 \mathrm{M}$ & 121 & 120 & $3 \mathrm{M}$ & 0 & $10 \mathrm{M}$ \\
STR2 & 3740 & 2.9 & $73 \mathrm{M}$ & 343 & 333 & $5 \mathrm{M}$ & 0 & 0 \\
MDD & 4974 & 25.2 & $73 \mathrm{M}$ & 457 & 425 & 0 & 0 & $28 \mathrm{M}$ \\
\hline
\end{tabular}

Table 5: Results of the propagators for instance set TSP-25 (times in seconds)

Tables 4 and 5 present the results. We first observe that STR2 and MDD perform worse than our propagators. AC5TC-Recomp is the winning strategy on tsp-20 instances while 
AC5TC-Tr is faster on the tsp-25 ones. The latter instances are more difficult. We can also see that checking the validity instead of the Q-validity allows AC5TC-Recomp to follow less pointers and perform fewer validity checks than the Q-validity checks of AC5TC-Bool and AC5TC-CutOff. Moreover, on these instances, the small arity makes the validity check $(O(r))$ cheap compared to Q-validity. Again, on those instances, the light-weight trailable structures of AC5TC-CutOff make it faster than AC5TC-Bool.

When we merge binary tables in tsp-20 instances into higher arity tables, we observed that AC5TC-Tr, our optimal algorithm, solves more instances than STR2, and with a smaller total execution time on the instances solved by both solvers. MDD does not compete on these instances. On simple instances, STR2 is more efficient than AC5TCRecomp which is also more efficient than AC5TC-Tr. However, on hard instances, the optimality of AC5TC-Tr pays off and it becomes the best algorithm.

Summary We conclude that, for the fully random instances, the lack of structure in the tables prevents our propagators from competing with state-of-the-art algorithms. However, for structured instances, our propagators are faster. Globally, AC5TC-Bool and AC5TC-CutOff are slower than AC5TC-Recomp since they are testing Q-validity, not validity, and hence they perform smaller jumps in the table. Moreover, maintaining their data structures is costly. Only the optimal AC5TC-Tr outperforms AC5TC-Recomp on difficult instances while using Q-validity. However, on easier instances, the cost of its trailable nextTr data-structure makes it slower than AC5TC-Recomp.

\section{Conclusion}

This paper proposed four different value-based, domain-consistency algorithms for table constraints, all using the AC5 generic framework. The new propagators record, for every value of the variables, the index of its first current support in the table. They also use, for each variable of a tuple, the index of the next tuple sharing the same value for this variable. They differ in their use of information on the validity of the tuples. AC5TC-Tr and AC5TC-Recomp are the two best value-based algorithms: AC5TCRecomp does not maintain any validity information and recomputes it on demand and AC5TC-Tr embeds the Q-validity information into the indexing structure, avoiding unnecessary visits of invalid tuples and leading to an optimal algorithm with a time complexity of $O(r \cdot t+r \cdot d)$ per table constraint. Our other algorithms have a time complexity of $O\left(r^{2} \cdot t+r \cdot d\right)$ per table constraint. Experimental results show that on, purely random tables, our algorithms do not compete with the state-of-the-art STR2+ and $\mathrm{MDD}^{c}$ algorithms. On structured instances, our propagators outperform STR2+ and MDD ${ }^{c}$, with a speed up varying between 1.83 and 4.57 . As future work, it would be interesting to extend AC5TC to handle negative tables through its disallowed tuples and to integrate the compressed representation of tuples introduced in [17].

Acknowledgments The authors want to thank the anonymous reviewers for their helpful comments. The first author is supported as a Research Assistant by the Belgian FNRS (National Fund for Scientific Research). This research is also partially supported by the Interuniversity Attraction Poles Program (Belgian State, Belgian Science Policy) and the FRFC project 2.4504.10 of the Belgian FNRS. 


\section{References}

1. C. Bessière and J.-C. Régin. Arc consistency for general constraint networks: Preliminary results. In IJCAI (1), pages 398-404, 1997.

2. M. Carlsson. Filtering for the case constraint. Talk given at the advanced school on global constraints, 2006

3. K. Cheng and R. Yap. An mdd-based generalized arc consistency algorithm for positive and negative table constraints and some global constraints. Constraints, 15:265-304, 2010.

4. Y. Deville and P. Van Hentenryck. Domain consistency with forbidden values. In D. Cohen, editor, Principles and Practice of Constraint Programming - CP 2010 - 16th International Conference, CP 2010, St. Andrews, Scotland, UK, September 6-10, 2010. Proceedings, volume 6308 of Lecture Notes in Computer Science, pages 191-205. Springer, 2010.

5. P. J. Fleming and J. J. Wallace. How not to lie with statistics: the correct way to summarize benchmark results. Commun. ACM, 29(3):218-221, Mar. 1986.

6. I. P. Gent, C. Jefferson, and I. Miguel. Watched literals for constraint propagation in minion. In Proceedings of CP2006, pages 182-197. Springer-Verlag, 2006.

7. I. P. Gent, C. Jefferson, I. Miguel, and P. Nightingale. Data structures for generalised arc consistency for extensional constraints. In Proceedings of the Twenty Second Conference on Artificial Intelligence, pages 191-197. AAAI Press, 2007.

8. G. Katsirelos and T. Walsh. A compression algorithm for large arity extensional constraints. In Proceedings of the 13th international conference on Principles and practice of constraint programming, pages 379-393. Springer-Verlag, 2007.

9. C. Lecoutre. Instances of the Constraint Solver Competition. http://www.cril.fr/ lecoutre/.

10. C. Lecoutre. Constraint Networks: Techniques and Algorithms. ISTE/Wiley, 2009.

11. C. Lecoutre. Str2: optimized simple tabular reduction for table constraints. Constraints, 16:341-371, 2011.

12. C. Lecoutre and R. Szymanek. Generalized arc consistency for positive table constraints. In Proceedings of CP 06, pages 284-298, 2006.

13. O. Lhomme. Arc-consistency filtering algorithms for logical combinations of constraints. In CPAIOR, pages 209-224, 2004.

14. O. Lhomme and J.-C. Régin. A fast arc consistency algorithm for n-ary constraints. In Proceedings of the Nationnal Conference on Artificial Intelligence, pages 405-410. AAAI Press, 2005.

15. L. Perron and V. Furnon. or-tools. http:// code.google.com/p/or-tools.

16. J.-C. Régin. Improving the expressiveness of table constraints. In Proceedings of workshop ModRef 11 at CP 11, 2011.

17. J.-C. Régin. Improving the expressiveness of table constraints. In In proceedings of ModRef'11 Workshop held with CP'11, 2011.

18. J. R. Ullmann. Partition search for non-binary constraint satisfaction. Inf. Sci., 177(18):3639-3678, 2007.

19. P. Van Hentenryck, Y. Deville, and C.-M. Teng. A generic arc-consistency algorithm and its specializations. Artif. Intell., 57(2-3):291-321, 1992.

20. P. Van Hentenryck and V. Ramachandran. Backtracking without Trailing in CLP( $\left(\Re_{l i n}\right)$. ACM Transactions on Programming Languages and Systems, 17(4):635-671, July 1995.

21. R. Wallace. Factor analytic studies of csp heuristics. In P. van Beek, editor, Principles and Practice of Constraint Programming - CP 2005, volume 3709, pages 712-726. Springer Berlin / Heidelberg, 2005.

22. K. Xu, F. Boussemart, F. Hemery, and C. Lecoutre. Random constraint satisfaction: Easy generation of hard (satisfiable) instances. Artif. Intell., 171(8-9):514-534, 2007. 